\title{
Vorwort zum III. Band
}

Der Scholz biegt mit Erscheinen des Bandes III in die Schlusskurve der 12. Auflage ein. Der Band enthält mit dem vierten, fünften und sechsten Abschnitt des $\mathrm{GmbH}-$ Gesetzes das Recht der Satzungsänderung, der Kapitalmaßnahmen, der Auflösung und der Liquidation unter Einschluss des immer bedeutsamer werdenden GmbH-Insolvenzrechts sowie des Ordnungs-, Straf- und Bußgeldrechts und des Einführungsgesetzes zum GmbH-Gesetz.

Die Verfasser verfolgen mit der Konzeption und Ausgestaltung des Kommentarwerks das Ziel, den täglichen Anforderungen wie auch den Zukunftsaufgaben einer wissenschaftlich fundierten Rechtspraxis gerecht zu werden. Neben zuverlässiger und gründlicher Information bietet der Kommentar deshalb durchgehend Vorschläge für die Beantwortung ungelöster Fragen durch Rechtsgestaltung und Judikatur. Garant dafür ist ein erfahrener Bearbeiterkreis, der sich auch im Band III weiter verjüngt hat. Herr Notar Dr. Joachim Tebben bearbeitet in Nachfolge von Herrn Notar a.D. Professor Dr. Hans-Joachim Priester das Recht der Satzungsänderungen ( $\$ \$ 53-59$ GmbHG). Herr Notarassessor Dr. Johannes Scheller (geb. Cziupka) hat die Auflösung ( $\$ \$ 60-62$ GmbHG) von Herrn Professor Dr. Dr. h.c. mult. Karsten Schmidt und Herrn Professor Dr. Georg Bitter und die Liquidation ( $\$ \$ 65-77$ GmbHG) von Herrn Professor Dr. Dr. h.c. mult. Karsten Schmidt übernommen. Herr Professor Dr. Georg Bitter hat mit der Übernahme des $₫ 64$ GmbHG das Insolvenzrecht der GmbH nun komplett in seinem Portfolio und Herr Professor Dr. Thomas Rönnau, der bereits in der 11. Auflage in die Fußstapfen von Herrn Professor Dr. Dr. h.c. mult. Klaus Tiedemann getreten war, ist nun allein verantwortlich für das gesamte GmbH-Strafrecht. Herr Professor Dr. André Meyer, der im Band II das Rechnungslegungsrecht von Herrn Professor Dr. Georg Crezelius übernommen hat, steuert sachgerecht die Kommentierungen der $\$ \$ 2,4,6,7$ EGGmbHG bei. Den ausgeschiedenen Autoren gilt auch an dieser Stelle unser allergrößter Dank für zuverlässige, tiefgängige und rechtsfortbildende Kommentierungen seit vielen Auflagen.

Der Generationenwechsel hat in weiten Teilen zu grundlegenden Überarbeitungen geführt. Im Einzelnen: Die Abschnitte zum Insolvenzrecht der $\mathrm{GmbH}$ sind in der 12. Auflage nochmals ausgebaut und teilweise neu gefasst worden. Damit liegt jetzt eine komplett neue, vorrangig die Gerichtspraxis des BGH und der OLG in den Blick nehmende Kommentierung des $\$ 64 \mathrm{GmbHG}$ aus der Feder von Professor Dr. Georg Bitter vor. Die ebenfalls von ihm verfasste Vorbemerkung zu $₫ 64 \mathrm{GmbHG}$ (Insolvenzgründe und Insolvenzverfahren) ist mit der Haftung wegen Masseschmälerung und Insolvenzverschleppung zu einem harmonischen Ganzen verschmolzen und um die Praxis besonders bewegende Fragen ergänzt, u.a. zur Beweislast im Prozess und zur Berücksichtigung streitiger Forderungen bei der Feststellung der Insolvenzreife. Die schon bisher größte Kommentierung der Gesellschafterdarlehen auf dem Markt im Anhang $\$ 64 \mathrm{GmbHG}$ wurde in der 12. Auflage in kritischer Analyse der aktuellen BGH-Rechtsprechung ebenfalls nochmals deutlich erweitert. Auch sie ist mit der Vorbemerkung zu $\$ 64$ GmbHG eng verzahnt, etwa in Bezug auf die aktuell diskutierten Fragen des (qualifizierten) Rangrücktritts. Als Hilfestellung in der aktuellen Corona-Krise finden sich am Ende der Kommentierungen des $\$ 64 \mathrm{GmbHG}$ und im Anhang $\$ 64$ GmbHG jeweils ausführliche Erläuterungen der einschlägigen Regelungen des COVID-19-Insolvenzaussetzungsgesetzes (COVInsAG). Kurz vor Drucklegung konnten hier sogar noch die im September beschlossene Änderung des COVInsAG berücksichtigt sowie Hinweise auf den RefE eines Gesetzes zur Fortentwicklung des Sanierungs- und Insolvenzrechts und den RefE einer Verordnung zur Verlängerung von Maßnahmen in Gesellschafts-, Genossenschafts-, Vereins- und Stiftungsrecht zur Bekämpfung der Auswirkungen der COVID-19-Pandemie aufgenommen werden.

Das gesamte Liquidationsrecht wurde in weiten Teilen von Herrn Notarassessor Dr. Johannes Scheller (geb. Cziupka) neu kommentiert und durchgehend auf den neuesten Stand gebracht. Es galt, eine Fülle obergerichtlicher, aber auch höchstrichterlicher, teils wegweisender Ent- 
scheidungen einzuarbeiten. Herausgegriffen seien hier nur die sich nun im neuen Lichte stellenden Fragen rund um die Fortsetzungsfähigkeit einer aufgelösten GmbH oder die amtswegige Löschung einer vermögenslosen $\mathrm{GmbH}$ (bei $\$ 60 \mathrm{GmbHG}$ ), und damit einhergehende, in der Praxis aber zuweilen überdehnte Möglichkeiten der Verkürzung des oftmals als lästig empfundenen Sperrjahres (bei $\$ \$ 60,73,74 \mathrm{GmbHG}$ ). Denen versucht die obergerichtliche Rechtsprechung zunehmend Einhalt zu gebieten, insbesondere sofern die (vorzeitige) Anmeldung des Liquidationsendes vor Abschluss des Steuerverfahrens erfolgt ( $\$ 74 \mathrm{GmbHG}$ ). Zahlreiche noch ungeklärte Folgefragen, die die höchstrichterliche Anerkennung eines Verfolgungsrechts übergangener Gläubiger gegenüber dem pflichtwidrig vor Sperrjahresablauf vermögenverteilenden Liquidator aufwirft, werden in das Haftungssystem eingeordnet und praxisgerechten Antworten zugeführt ( $\$ 73 \mathrm{GmbHG})$. Ebenfalls durchgängig eingearbeitet ist die Liquidation der GmbH \& Co. KG. Einen weiteren Schwerpunkt bilden die Nachtragsliquidation ( $\$ \$ 60,66,74 \mathrm{GmbHG}$ ), die in der Praxis oftmals Probleme bereitet, wie zahlreiche neuere Gerichtsentscheidungen belegen, sowie die Frage nach der Reichweite des Diskontinuitätsgedankens bzgl. der Vertretungsbefugnis der Liquidatoren ( $\$ 68 \mathrm{GmbHG})$ mitsamt ihren Implikationen für die Handelsregisteranmeldung ( $\$ 67 \mathrm{GmbHG})$.

Auch das GmbH-Strafrecht wartet mit einigen Neuerungen auf. Wegen der Neufassung von $\$ 15$ a Abs. 4-6 InsO zum 26.6.2017 in Reaktion auf eine vielfach beklagte „Überkriminalisierung" der Insolvenzverschleppung in der Tatvariante des Nicht-richtig-Stellens des Eröffnungsantrags hat Herr Professor Dr. Thomas Rönnau in der Kommentierung Vor $\$ \$ 82 \mathrm{ff}$. GmbHG erläutert, welche strafrechtlichen Risiken in diesem Zusammenhang trotz Einführung einer strafbarkeitseinschränkenden objektiven Strafbarkeitsbedingung noch bestehen. Meinungsbildend befasst sich Herr Rönnau zudem mit dem zum 26.4.2019 in Kraft getretenen GeschGehG und seiner Bedeutung für $\$ 85 \mathrm{GmbHG}$ (Anwendbarkeit, etwaige Veränderungen beim Geschäftsgeheimnisbegriff). Erstmals kommentiert werden im Scholz zudem die durch das AReG neu geschaffenen $\$ \$ 86-88$ GmbHG, die sich mit der Straf- und Bußbarkeit von Pflichtverletzungen bei Abschlussprüfungen beschäftigen. Last but not least erwähnenswert ist die aktualisierte, alphabetisch aufgelistete Rechtsprechung zur GmbH-Untreue (Vor $\$ \$ 82$ ff. GmbHG Rz. 16).

Insgesamt befindet sich der III. Band auf dem Stand von September 2020. Eine Vielzahl der in diesem Band enthaltenen Kommentierungen wurde schon während des Herstellungsprozesses des Printbandes in die Datenbanken, in denen der Scholz enthalten ist, vorab eingestellt. Durch diese Online-first-Strategie konnte zum Nutzen der Datenbank-Abonnenten eine besondere Aktualität erzielt werden. Vor Drucklegung wurden diese Inhalte von den Autoren nochmals aktualisiert, so dass der Band auf einheitlichem Stand ist.

Den Aktualitätsvorteil der Online-first-Strategie wollen die Scholz-Autoren und der Verlag auch zukünftig nutzen und planen daher, bei besonderem Anlass (vor allem grundlegenden Gesetzesänderungen) den Austausch ganzer Kommentierungen in der Online-Version auch unabhängig von der Printveröffentlichung fortzusetzen. Es lohnt daher - sofern nicht schon geschehen - eine Beschäftigung mit der Online-Version des Scholz. Aktuell ist der Scholz in mehreren Datenbanken vertreten - bei Juris im PartnerModul GmbH-Recht und im Zusatzmodul für die Hochschulen, bei Otto Schmidt online im Aktionsmodul Gesellschaftsrecht und Beratermodul Kommentare Gesellschaftsrecht (weitere Informationen dazu unter www. juris.de/pm-gmbhrecht und www.otto-schmidt.de/akgr).

Verschiedentlich erreichen uns Hinweise aus dem Kreis der Benutzer, die wir gerne aufgreifen und berücksichtigen. Wir bitten unsere Leser, uns auch künftig in gleicher Weise zu unterstützen. Anregungen und Bemerkungen können gerne per E-Mail (lektorat@otto-schmidt.de) an den Verlag geschickt werden. 
Es bearbeiten im III. Band:

Georg Bitter

Vor $₫ 64$ Insolvenz der GmbH und der GmbH \& Co. KG, $₫ 64$ (in Nachfolge Karsten Schmidt), Anhang $\$ 64$ Gesellschafterdarlehen

André Meyer $\$ \S 2,4,6,7$ EGGmbHG

Thomas Rönnau

Vor $\$ \$ 82 \mathrm{ff}$. GmbH und Strafrecht, $\$ \$ 82-88$

Johannes Scheller $\$ \$ 60-62$ (in Nachfolge Karsten Schmidt und Georg Bitter), (geb. Cziupka) $\$ \$ 65-70,72-77$ (in Nachfolge Karsten Schmidt)

Karsten Schmidt $\quad \$ 71$

Uwe H. Schneider

$\S 1$ EGGmbHG

Christoph H. Seibt $\S \$ 3,8$ EGGmbHG

Joachim Tebben $\$ \$ 53-59$ (in Nachfolge Hans-Joachim Priester)

Hartmut Wicke $\$ \$ 78,79$ 
